\title{
Encyclopedia
}

\section{DNA Excision Repairs and Implication on Human Health}

Created by: Tae-hong Kang

Version received: 13 December 2019

check for

updates

The physiological impact of the aberrant oxidation products on genomic DNA were demonstrated by embryonic lethality or the cancer susceptibility and/or neurological symptoms of animal impaired in the base excision repair (BER); the major pathway to maintain genomic integrity against non-bulky DNA oxidation. However, growing evidence suggests that other DNA repair pathways or factors that are not primarily associated with the classical BER pathway are also actively involved in the mitigation of oxidative assaults on the genomic DNA, according to the corresponding types of DNA oxidation. Among others, factors dedicated to lesion recognition in the nucleotide excision repair (NER) pathway have been shown to play eminent roles in the process of lesion recognition and stimulation of the enzyme activity of some sets of BER factors. Besides, substantial bulky DNA oxidation can be preferentially removed by a canonical NER mechanism; therefore, loss of function in the NER pathway shares common features arising from BER defects, including cancer predisposition and neurological disorders, although NER defects generally are nonlethal.

DNA lesions arising from the ROS attack can generate both non-bulky (non-helix distorting) and bulky (helix distorting) lesions. In human cells, BER and NER are the two DNA excision repair pathways responsible for the removal of nonbulky and bulky DNA lesions, respectively. Both repair pathways share three common steps, which include 1) lesion recognition, 2) excision of damaged nucleotide, and 3) resynthesis using error-free DNA polymerases (Figure 1).

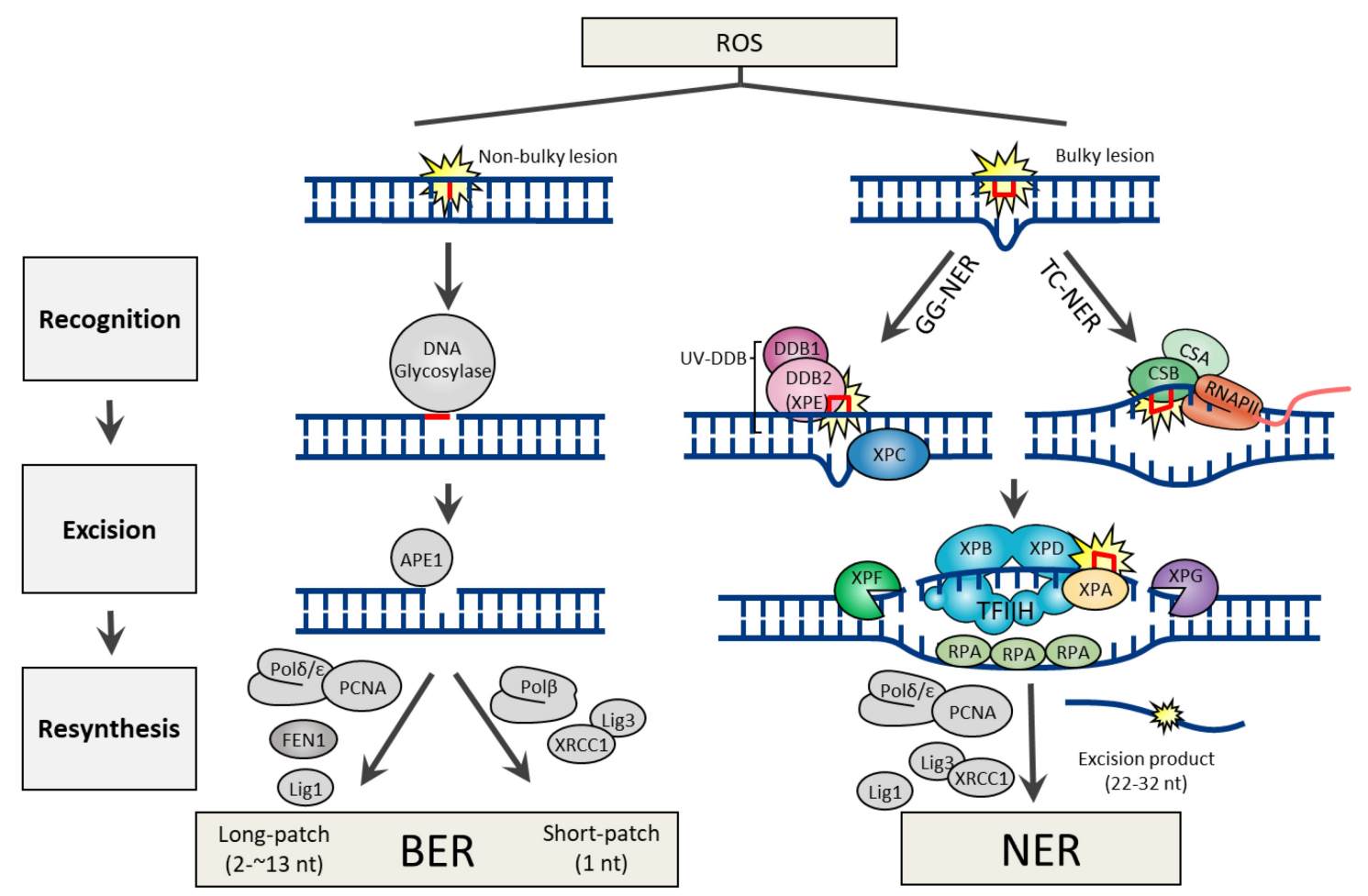

Figure 1. DNA excision repair mechanisms for bulky or non-bulky DNA lesions caused by reactive oxygen species (ROS).

\section{Base Excision Repair}

Many of the genes involved in BER are highly conserved from bacteria to humans ${ }^{[1]}$, indicating that BER is a fundamental repair pathway in most living organisms. The BER pathway is specialized to fix non-bulky single-base lesions in the form of small chemical modifications, including oxidation, alkylation, and deamination damage. Base 
modifications are pro-mutagenic and/or cytotoxic, depending on how they interfere with the template function of the DNA during replication and transcription. To initiate the procedure, BER employs a specific DNA glycosylase for lesion recognition and elimination of the damaged base. Although every DNA glycosylase has a distinct structure and substrate specificity, all glycosylases share a common mode of action for damage recognition; 1) flipping the affected base out of the DNA helix, which facilitates a sensitive detection of even minor base modifications, 2) catalyzing the cleavage of an $\mathrm{N}$-glycosidic bond, releasing a free base and creating an abasic site (apurinic/apyrimidinic site or AP site). DNA glycosylases can be either monofunctional or bifunctional. Monofunctional DNA glycosylases possess only the glycosylase activity, which includes UNG (uracil-N glycosylase), SMUG1 (single-strand-specific monofunctional uracil DNA glycosylase), MBD4 (methyl-binding domain glycosylase 4), TDG (thymine DNA glycosylase), MYH (MutY homolog DNA glycosylase), MPG (methylpurine glycosylase). In contrast, bifunctional DNA glycosylases have an intrinsic 3' AP lyase activity accompanying with the glycosylase activity, which includes OGG1 (8-oxoguanine DNA glycosylase), NTH1 (endonuclease III-like), and NEIL1 (endonuclease VIII-like glycosylase). The processes following DNA glycosylase are common to the BER mechanism irrespective of the identity of the glycosylase. Base removal by a DNA glycosylase generates an AP site in DNA, which is then further processed by AP endonuclease 1 (APE1), which cleaves the DNA backbone 5' to the abasic site, generating a 3'-hydroxyl and a 5'-2-deoxyribose-5'-phosphate (5'-dRP). DNA polymerase $\beta$ (Pol $\beta$ ) utilizes the 3'-hydroxyl to fill the gap through template-directed synthesis. Depending on the number of nucleotides added, either short-patch (where a single nucleotide is replaced) or long patch BER (where 2-13 nucleotides are synthesized) pathways operate to complete the repair process. In short-patch BER, the intrinsic dRP-lyase activity of Pol $\beta$ removes the $5^{\prime}$ - $d R P$. The addition of more than one nucleotide (up to 13) constitutes long-patch BER and requires the assistance of flap endonuclease 1 (FEN1) to remove the displaced 5'-flap structure (Figure 1).

\section{General Features of BER Defect}

Genetic loss or mutation in key genes of the BER process, such asAPE1 ${ }^{[2]}$, Pol $\beta^{[3]}$, FEN1 ${ }^{[4]}$, or the DNA ligase $3^{[5]}$, has been shown to have embryonic lethality in mice, while the phenotype of DNA glycosylase disruptions in mice is usually rather moderate, the only known exception being the TDG, which was essential for embryonic development in mice [6][7].

Neurons encounter particularly high levels of oxidative stress because of the high metabolic rate required to support their electrical and synaptic functions. Thus, the integrity and capacity of systems that repair oxidative DNA damage would be expected to be critical for the survival and proper function of neurons, particularly under conditions of increased oxidative stress that occur during catastrophic pathological conditions, including ischemic stroke. In a study with a mouse model of focal cerebral ischemic brain injury performed on normal and OGG1 $1^{-/}$mice, the potential role of OGG1 in ameliorating the detrimental effect of oxidative DNA damage to neurons was evaluated. The results indicate that after cerebral ischemia, the accumulation of brain oxidative DNA base lesions was significantly greater in OGG1-deficient mice, and was associated with greater brain damage and poorer behavioral outcomes, revealing an important role for OGG1 in brain BER capacity, which contributes to neuronal survival after experimental stroke ${ }^{[8]}$.

Neurons from OGG1-deficient mice are sensitive to oxidative stress and reduced OGG1 levels have also been associated with Alzheimer's disease (AD) [9]. Indeed, levels of expression of UNG, OGG1, and Pol $\beta$ are lower in brain tissue from patients with $A D$ than in brain tissue from age-matched controls without AD [10]. Po/ $\beta^{+/-}$heterozygote displayed impaired synaptic and cognitive functions, linking the loss of heterozygous BER function in the progression of AD [11]

While a mouse lacking UNG develops B-cell lymphomas, there is often not a clear phenotype in a mouse for a single DNA glycosylase mutation, presumably due to the substrate redundancy. For instance, the OGG1-deficient mice are viable and fertile without any visible phenotype [12][13], which suggests overlapping activities for the repair of 8-oxoG lesions. However, interestingly, additional deletion of $M Y H$ in OGG1-deficient mice predisposes $65.7 \%$ of mice to tumors, predominantly lung and ovarian tumors, and lymphomas [14]. 8-oxoG indeed is a common substrate of MYH and OGG1.

\section{Nucleotide Excision Repair}

NER is famous for the unique repair pathway in humans to remove photolesions produced by UV radiation (sun exposure) that mainly forms cyclobutane pyrimidine dimer (CPD), a non-bulky lesion and pyrimidine-(6,4)-pyrimidone 
product (6-4PP), a bulky lesion. Besides, it also efficiently eliminates an extremely broad range of structurally unrelated DNA lesions, including bulky chemical adducts and intrastrand crosslinks [15]. The basis of the versatility of NER originates that it circumvents recognition of the lesion itself, instead, the lesion recognizing NER factors detects the presence of unpaired single-stranded DNA opposite the damaged strand [16].

Owing to the distinct damage recognition events, NER mechanisms can be further specified into two subpathways, global genome NER (GG-NER) and transcription-coupled NER (TC-NER). The former is responsible for eliminating lesions throughout the whole genome, while the latter is for those in the transcribing strand of active genes. During GGNER, XPC (xeroderma pigmentosum C) or UV-DDB (UV-damaged DNA binding protein; a heterodimeric complex with DDB1 and DDB2) initiate the recognition of the damage. Structural analysis showed certain lesions, such as CPD, which do not significantly distort the DNA helix, are first recognized by DDB2 (also known as XPE) to extrude the lesions into its binding pocket, and thereby create a kink that is now recognized by XPC [17]. If the lesion is in a transcribed gene, it is sensed as a blockage to RNA polymerase II (RNAPII) and requires Cockayne syndrome B (CSB) and CSA to initiate the TC-NER process [18]. Regardless of the damage recognition mechanisms, the downstream events are conserved in both NER mechanisms. Damage verification is executed by XPA and helix unwinding is carried out by TFIIH (complexed with the XPB and XPD helicases). Lesion excision is catalyzed by the structure-specific endonucleases XPF and XPG, which incise the damaged strand at $5^{\prime}$ and $3^{\prime}$ from the lesion, respectively, which promotes releasing out of the lesion containing 22-32 nt-long oligomers. Final DNA gap-filling synthesis and ligation are executed by the replication proteins

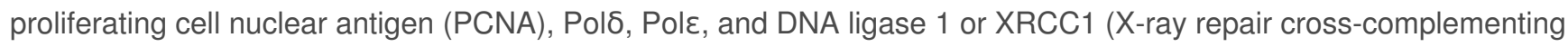
protein 1)-DNA ligase 3 complex (Figure 1).

\section{General Features of NER Defect}

Hereditary mutations in NER-associated genes are nonlethal and associated with disorders that are characterized by UV sensitivity and cancer predisposition, such as XP, CS, and trichothiodystrophy (TTD) [19]. XP comprises seven complementation groups ( $X P A-X P G$ ) with defective GG-NER. Five of these groups also exhibit defective TC-NER, whereas XP-C and XP-E patients are TC-NER-proficient. The XP patient shows hypersensitivity on minimal sun exposure, pigmentary accumulations at exposed skin regions, and multiple early age skin cancers. Progressive neuronal degeneration is also observed in approximately one-third of XP cases, generally after the appearance of cutaneous signs [20]. However, unlike GG-NER-deficient XP patients, CS patients belong to one of two complementation groups CS-A or $C S-B)$, and those that are completely defective in TC-NER are not cancer-prone but exhibit a drastic reduction in life span [21]. In addition, CS patients display a number of neurological and developmental abnormalities as well as hypersensitivity to sun exposure. Although the molecular basis that leads to the diverse features of CS remains largely unknown, a reduced ability of cells to relieve oxidative stress has been proposed to be a leading cause [2][23][24]. Since cells from CS patients were found to be hypersensitive to oxidative DNA damage, a role for the CS proteins in the response to oxidized bases has been proposed [25]. Mutations in the CSB account for the majority of CS cases $\underline{[26]}$.

Clinical heterogeneity in disorders with NER mutations opens the question of whether defects in this pathway are solely due to impaired repair of helix-distorting DNA lesions. XP patients along with TCR defects (caused by some specific alterations in $X P B, X P D$, and $X P G$ ) present, besides increased skin cancer risk, accelerated neurodegeneration, and CS symptoms (XP/CS). The causative relationship between mutations and the CS clinical features in XP/CS cases is complex and must not only involve the NER defects but also the other functions of the NER proteins. Several studies have demonstrated that transcription impairment, oxidative repair, and energy metabolism alteration, as well as genotoxic stress, may explain the combined XP/CS phenotype [27][28]. Neuronal death might be due to accumulated endogenous damage, and indeed a growing body of evidence indicates that NER proteins participate in the processing of oxidative DNA lesions that are produced by the normal cell metabolism. The role of NER proteins in different pathways might explain the heterogeneity in disorders with NER mutations [29].

The publication can be found here: https://www.mdpi.com/1422-0067/20/23/6092/htm

\section{References}


1. A. B. Robertson; A. Klungland; Torbjørn Rognes; I. Leiros; DNA repair in mammalian cells: Base excision repair: the long and short of it. Cellular and Molecular Life Sciences 2009, 66, 981-993, 10.1007/s00018-009-8736-z.

2. S. Xanthoudakis; R J Smeyne; J. D. Wallace; Tom Curran; The redox/DNA repair protein, Ref-1, is essential for early embryonic development in mice.. Proceedings of the National Academy of Sciences 1996, 93, 8919-8923, 10.1073/pnas.93.17.8919.

3. H Gu; J. Marth; P. Orban; H Mossmann; Klaus Rajewsky; Deletion of a DNA polymerase beta gene segment in T cells using cell typespecific gene targeting. Science 1994, 265, 103-106, 10.1126/science.8016642.

4. Melanie Kucherlapati; Kan Yang; Mari Kuraguchi; Jie Zhao; Maria Lia; Joerg Heyer; Michael F. Kane; Kunhua Fan; Robert Russell; Anthony M. C. Brown; et al.Burkhard KneitzWinfried EdelmannRichard D. KolodnerMartin LipkinRaju Kucherlapati Haploinsufficiency of Flap endonuclease (Fen1) leads to rapid tumor progression. Proceedings of the National Academy of Sciences 2002, 99, 9924-9929, 10.1073/pnas.152321699.

5. Nahum Puebla-Osorio; Devin B. Lacey; Frederick W. Alt; Chengming Zhu; Early Embryonic Lethality Due to Targeted Inactivation of DNA Ligase III†. Molecular and Cellular Biology 2006, 26, 3935-3941, 10.1128/MCB.26.10.3935-3941.2006.

6. Daniel Cortázar; Christophe Kunz; Jim Selfridge; Teresa Lettieri; Yusuke Saito; Eilidh MacDougall; Annika Wirz; David Schuermann; Angelika L. Jacobs; Fredy Siegrist; et al.Roland SteinacherJosef JiricnyAdrian BirdPrimo Schär Embryonic lethal phenotype reveals a function of TDG in maintaining epigenetic stability. Nature 2011, 470, 419-423, 10.1038/nature09672.

7. Salvatore Cortellino; Jinfei Xu; Mara Sannai; Robert Moore; Elena Caretti; Antonio Cigliano; Madeleine Le Coz; Karthik Devarajan; Andy Wessels; Dianne Soprano; et al.Lara K. AbramowitzMarisa S. BartolomeiFlorian RambowMaria Rosaria BassiTiziana BrunoMaurizio FanciulliCatherine RennerAndres J. Klein-SzantoYoshihiro MatsumotoMinique Kobilrwin DavidsonChristophe AlbertiLionel LaRueAlfonso Bellacosa Thymine DNA glycosylase is essential for active DNA demethylation by linked deamination-base excision repair.. Cell 2011, 146, 67-79, 10.1016/j.cell.2011.06.020.

8. Ng Liu; Deborah L Croteau; Nadja Souza-Pinto; Michael Pitta; Jingyan Tian; Christopher Wu; Haiyang Jiang; Khadija Mustafa; Guido Keijzers; Vilhelm A Bohr; et al.Mark P Mattson Evidence that OGG1 glycosylase protects neurons against oxidative DNA damage and cell death under ischemic conditions. British Journal of Pharmacology 2010, 31, 680-692, 10.1038/jcbfm.2010.147.

9. Meryl S. Lillenes; Alberto Rabano; Mari Støen; Tahira Riaz; Dorna Misaghian; Linda Møllersen; Ying Esbensen; Clara-Cecilie Günther; Per Selnes; Vidar T. V. Stenset; et al.Tormod FladbyTone Tønjum Altered DNA base excision repair profile in brain tissue and blood in Alzheimer's disease. Molecular Brain 2016, 9, 61, 10.1186/s13041-016-0237-z.

10. Lior Weissman; Ng-Gyu Jo; Martin M. Sørensen; Nadja C. De Souza-Pinto; William R. Markesbery; Mark P. Mattson; Vilhelm A. Bohr; Defective DNA base excision repair in brain from individuals with Alzheimer's disease and amnestic mild cognitive impairment.. Nucleic Acids Research 2007, 35, 5545-55, 10.1093/nar/gkm605.

11. Peter Sykora; Magdalena Misiak; Yue Wang; Somnath Ghosh; Giovana S. Leandro; Ng Liu; Jane Tian; Beverly A. Baptiste; Wei-Na Cong; Boris M. Brenerman; et al.Evandro FangKevin G. BeckerRoyce J. HamiltonSoumya ChigurupatiYongqing ZhangJosephine M. EganDeborah L. CroteauDavid M. WilsonMark P. MattsonVilhelm A. Bohr DNA polymerase $\beta$ deficiency leads to neurodegeneration and exacerbates Alzheimer disease phenotypes.. Nucleic Acids Research 2014, 43, 943-59, 10.1093/nar/gku1356.

12. Arne Klungland; Ian Rosewell; Stephan Hollenbach; Elisabeth Larsen; Graham Daly; Bernd Epe; Erling Seeberg; Tomas Lindahl; Deborah E. Barnes; Accumulation of premutagenic DNA lesions in mice defective in removal of oxidative base damage. Proceedings of the National Academy of Sciences 1999, 96, 13300-13305, 10.1073/pnas.96.23.13300.

13. Osamu Minowa; Tsuyoshi Arai; Masanori Hirano; Yoshiaki Monden; Shigeyasu Nakai; Mayuko Fukuda; Masaki Itoh; Hiroshi Takano; Yoshitaka Hippou; Hiroyuki Aburatani; et al.Ken-Ichi MasumuraTakehiko NohmiSusumu NishimuraTetsuo Noda Mmh/Ogg1 gene inactivation results in accumulation of 8-hydroxyguanine in mice. Proceedings of the National Academy of Sciences 2000, 97, 41564161, 10.1073/pnas.050404497.

14. Yali Xie; Hanjing Yang; Cristina Cunanan; Kimberly Okamoto; Darryl Shibata; Janet Pan; Deborah E. Barnes; Tomas Lindahl; Michael Mcllhatton; Richard Fishel; et al.Jeffrey H. Miller Deficiencies in mouse Myh and Ogg1 result in tumor predisposition and G to T mutations in codon 12 of the K-ras oncogene in lung tumors.. Cancer Research 2004, 64, 3096-3102, 10.1158/0008-5472.CAN-033834.

15. Jurgen A. Marteijn; Hannes Lans; Wim Vermeulen; Jan H. J. Hoeijmakers; Understanding nucleotide excision repair and its roles in cancer and ageing. Nature Reviews Molecular Cell Biology 2014, 15, 465-481, 10.1038/nrm3822.

16. Masayuki Kusakabe; Yuki Onishi; Haruto Tada; Fumika Kurihara; Kanako Kusao; Mari Furukawa; Shigenori Iwai; Masayuki Yokoi; Wataru Sakai; Kaoru Sugasawa; et al. Mechanism and regulation of DNA damage recognition in nucleotide excision repair.. Genes and Environment 2019, 41, 2, 10.1186/s41021-019-0119-6.

17. Andrea Scrima; Renata Koníčková; Bryan K. Czyzewski; Yusuke Kawasaki; Philip D. Jeffrey; Regina Groisman; Yoshihiro Nakatani; Shigenori Iwai; Nikola P. Pavletich; Nicolas H. Thoma; et al. Structural basis of UV DNA-damage recognition by the DDB1-DDB2 complex.. Cell 2008, 135, 1213-23, 10.1016/j.cell.2008.10.045.

18. Hannes Lans; Jan H. J. Hoeijmakers; Wim Vermeulen; Jurgen A. Marteijn; The DNA damage response to transcription stress. Nature Reviews Molecular Cell Biology 2019, 20, 766-784, 10.1038/s41580-019-0169-4.

19. Jeong-Min Park; Tae-Hong Kang; Transcriptional and Posttranslational Regulation of Nucleotide Excision Repair: The Guardian of the Genome against Ultraviolet Radiation. International Journal of Molecular Sciences 2016, 17, 1840, 10.3390/ijms17111840.

20. Alan R Lehmann; David McGibbon; Miria Stefanini; Xeroderma pigmentosum. Orphanet Journal of Rare Diseases 2011, 6, 70-70, 10.1186/1750-1172-6-70. 
21. Yuming Wang; Probir Chakravarty; Michael Ranes; Gavin Kelly; Philip J. Brooks; Edward Neilan; Aengus Stewart; Giampietro Schiavo; Jesper Q. Svejstrup; Dysregulation of gene expression as a cause of Cockayne syndrome neurological disease.. Proceedings of the National Academy of Sciences 2014, 111, 14454-9, 10.1073/pnas.1412569111.

22. Barbara Pascucci; Tiziana Lemma; Egidio lorio; Sara Giovannini; Bruno Vaz; Ivano lavarone; Angelo Calcagnile; Laura Narciso; Paolo Degan; Franca Podo; et al.Vera RoginskyaBratislav M. JanjicBennett Van HoutenMiria StefaniniEugenia DogliottiMariarosaria D’Errico An altered redox balance mediates the hypersensitivity of Cockayne syndrome primary fibroblasts to oxidative stress. Aging Cell 2012 , 11, 520-529, 10.1111/j.1474-9726.2012.00815.x.

23. Luciana Nogueira De Sousa Andrade; Jason L. Nathanson; Gene W. Yeo; Carlos Frederico Martins Menck; Alysson Renato Muotri; Evidence for premature aging due to oxidative stress in iPSCs from Cockayne syndrome. Human Molecular Genetics 2012, 21, 38253834, 10.1093/hmg/dds211.

24. James E. Cleaver; Vladimir Bezrookove; Ingrid Revet; Eric J. Huang; Conceptual developments in the causes of Cockayne syndrome. Mechanisms of Ageing and Development 2013, 134, 284-290, 10.1016/j.mad.2013.02.005.

25. Tinna Stevnsner; Meltem Muftuoglu; Maria Diget Aamann; Vilhelm A. Bohr; The role of Cockayne Syndrome group B (CSB) protein in base excision repair and aging.. Mechanisms of Ageing and Development 2008, 129, 441-8, 10.1016/j.mad.2008.04.009.

26. Christine Troelstra; Alain Van Gool; Jan De Wit; Wim Vermeulen; Dirk Bootsma; Jan H.J. Hoeijmakers; ERCC6, a member of a subfamily of putative helicases, is involved in Cockayne's syndrome and preferential repair of active genes. Cell 1992, 71, 939-953, 10.1016/0092-8674(92)90390-x.

27. Anneke Van Hoffen; Wouter H. J. Kalle; Astrid De Jong-Versteeg; Alan R. Lehmann; Albert A. Van Zeeland; Leon H. F. Mullenders; Cells from XP-D and XP-D-CS patients exhibit equally inefficient repair of UV-induced damage in transcribed genes but different capacity to recover UV-inhibited transcription.. Nucleic Acids Research 1999, 27, 2898-2904, 10.1093/nar/27.14.2898.

28. Daniela Tathiana Soltys; Clarissa R. R. Rocha; Letícia K. Lerner; Tiago A. De Souza; Veridiana Munford; Fernanda Cabral; Tiziana Nardo; Miria Stefanini; Alain Sarasin; Januário B. Cabral-Neto; et al.Carlos F. M. Menck NovelXPG(ERCC5) Mutations AffectDNARepair and Cell Survival after Ultraviolet but not Oxidative Stress. Human Mutation 2013, 34, 481-489, 10.1002/humu.22259.

29. B. Pascucci; M. D’Errico; E. Parlanti; S. Giovannini; E. Dogliotti; Role of nucleotide excision repair proteins in oxidative DNA damage repair: an updating.. Biochemistry (Moscow) 2010, 76, 4-15, 10.1134/s0006297911010032.

\section{Keywords}

Nucleotide excision repair; Base excision repair; DNA oxidation; ROS (reactive oxygen species); Genetic disorders; Human health (C) 2019 by the author(s). Distribute under aCreative Commans CC BY license 